\title{
Computational model of the deformation of graded wedge with complicated structure
}

\author{
Irina S. Trubchik*, Ludmila N. Evich, and Evgenii N. Ladosha \\ Don State Technical University, Department of Mass media and multimedia technologies, 344000 \\ Rostov-on-Don Gagarina 1, Russia
}

\begin{abstract}
As a computational model of deformation of wedge-shaped gradient coatings lying on a non-deformable base, we propose a solution of the contact problem for a wedge non-uniform in thickness rigidly fixed on the lower face. A closed analytic solution of the contact problem is obtained for the case of elastic moduli (Young's modulus, Poisson's ratio, shear modulus), which is a continuous differentiable function with respect to the angular coordinate. The influence of the gradient of the wedge on the distribution of contact stresses under the stamp is illustrated. The problem is posed in connection with the need to predict the phenomenon of delamination of modern coatings of a complex structure from a nondeformable substrate.
\end{abstract}

\section{General problem statement}

The static contact problem for an elastic wedge-shaped region with opening angle $\alpha(0 \leq \varphi \leq \alpha)$ is considered. The cylindrical coordinate system $(r, \varphi, z)$ is associated with this area. We assume that the face of the wedge $\varphi=0$ is rigidly fixed, and unyielding flat stamp acts on the brink of $\varphi=\alpha$. Per unit length of the stamp act shear force $P \mathrm{c}$, parallel to the axis $z$, the action of which the stamp is moved in the direction of the z-axis by an amount $\varepsilon$, causing the wedge deformation of pure shear (problem $W 1$ ); or stamp is pressed into the wedge by the power $\mathrm{P}$ (here the force assigned to the unit length of the die) is applied at a distance $\mathrm{H}$ from the vertex the wedge (the problem $W 2$ ). Let's assume that the friction force between the stamp and the surface of the wedge is absent and surface of the wedge $\varphi=\alpha$ is not loaded the out of stamp. The width of the contact area of the stamp with a wedge defined by the inequality $a \leq r \leq b$, and base form of the stamp in contact zone by the function $\gamma(r)$ (Fig. 1). For both problems the Poisson's ratio $v=$ const, the shear modulus and Young's modulus of the wedge vary according to the law

$$
G=G_{\alpha} f(\varphi), E=E_{\alpha} f(\varphi), v=v_{\alpha} f(\varphi), 0 \leq \varphi \leq \alpha
$$

where $f(\varphi)$ - arbitrary positive definite, continuously differentiable function everywhere in the domain. The gradient changes of elastic properties of the wedge can be positive, negative or change sign for $0 \leq \varphi \leq \alpha$.

\footnotetext{
* Corresponding author: trubchik@mail.ru
} 
W1

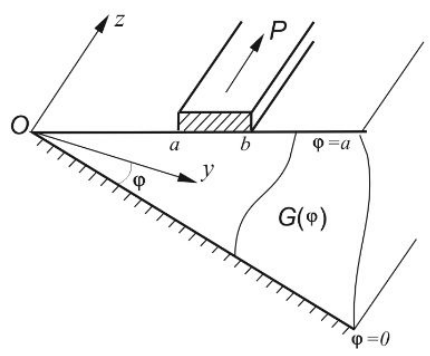

W2

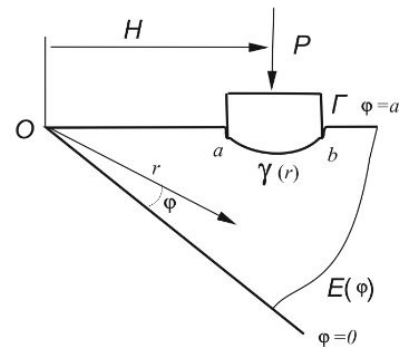

Fig. 1. Schemes of contact problems for the inhomogeneous wedge

The boundary conditions of the problem under these assumptions are of the form:

$$
(W 1): w(r, 0, z)=0,(\mathrm{~W} 2): u(r, 0)=v(r, 0)=0, a \leq r \leq b, \varphi=0
$$

$W 1: \tau_{\varphi_{Z}}(r, \alpha, z)=0(0<r<a, r>b) ; \quad w(r, \alpha, z)=\varepsilon(a \leq r \leq b)$

$W 2: \tau_{r \varphi}(r, \alpha)=0, \sigma_{\varphi}(r, \alpha)=0(0<r<a, r>b) ; v(r, \alpha)=-\delta(r)(a \leq r \leq b)$

Required to determine the distribution of tangents contact (for $W 1$ problem) and normal contact (for $W 2$ problem) stresses under the stamp

$$
(W 1): \tau_{\varphi z}(r, \alpha, z)=-\tau(r),(W 2): \phi(r, \alpha)=-q(r) \quad(a \leq r \leq b)
$$

\section{Computational model building}

\subsection{Reducing the problems for the inhomogeneous wedge to solving of the integral equation (IE)}

The problems $(W 1)$ and $(W 2)$ reduce to the solution of the Fredholm integral equation with the logarithmic kernel

$$
\int_{-1}^{1} \phi(\xi) k\left(\frac{\xi-x}{\lambda}\right) d \xi=2 \pi C_{0} g\left(x^{\prime}\right),\left|x^{\prime}\right| \leq 1, k(t)=\int_{0}^{\infty} L(\alpha) \alpha^{-1} \cos (\alpha t) d \alpha
$$

where $\quad k(t)=\int_{0}^{\infty} L_{1}(u, \alpha) u^{-1} \cos (u t) d u, \quad t=\ln (\rho / r), \quad \xi=\lambda \ln (\rho / a)-1$, $x=\lambda \ln (r / a)-1, \lambda=2(\ln (b / a))^{-1}, \phi(\xi)$ - contact stress distribution function, $\theta_{\alpha}=G_{\alpha} /(1-v), G_{\alpha}=G(\varepsilon)$ - shear modulus at the top surface of the wedge.

Here $\phi(\xi)=\rho \tau(\rho), g(x)=G_{\alpha} \lambda \varepsilon$ (for problem W1), $\phi(\xi)=\rho q(\rho), g(x)=G_{\alpha} \lambda \varepsilon$ (for problem W2). The dimensionless parameter $\lambda(0<\lambda<\infty)$ characterizes the relative position of the stamp on the wedge face. If the parameter $\lambda$ is large (small), then the stamp is located relatively far (close) from the top of the elastic wedge. We note that the integral equation (5) for $\alpha \rightarrow 0$ and fixed $H=a g \alpha$ degenerates into an integral equation of the corresponding contact problems for an elastic layer (strip) [1].

To complete the formulation of the contact problems examined for an elastic inhomogeneous wedge to the integral equation (5), the equilibrium condition of the stamp

$$
(W 1): P_{c}=\int_{a}^{b} \tau(r) d r,(W 2): P=\int_{a}^{b} q(r) d r
$$

which (with $\alpha=\pi$ and $\alpha=2 \pi$ ) makes it possible to determine the relationship between the shearing force and the displacement of the stamp $\mathrm{P}_{\varepsilon}$ and the displacement of the stamp $\varepsilon(\mathrm{W} 1)$ 
or the pressing force $P$ and the displacement of the stamp $\delta$ (W2). If the problem statement is correct, condition $\tau(r) \geq 0$ or $\sigma(r) \geq 0$ for $a \leq r \leq b$ should be satisfied.

\subsection{Numerical construction of the IE transforms functions (Modulating functions method)}

The method of integral transformations and generalization of method for the simulating functions is used for the numerical construction of transformant kernels of integral equations of the coordinate systems other than Cartesian [2]. The problems are reduced to the solution of ordinary differential equations of second order (in antiplane problem $W 1$ ) or a system of second order differential equations with variable coefficients (in plane problem W2) relatively the integral transforms of the Mellin transform of the displacement.

$$
\frac{d \overrightarrow{\mathbf{w}}(u, \varphi)}{d \varphi}=\mathbf{A} \overrightarrow{\mathbf{w}}(u, \varphi), \varphi \in[0, \alpha]
$$

In the problem of $W 1$ rank of matrix $\mathrm{A}$ is equal to two, and the problem of $W 2$ - equal to four. The entries of matrix are the variables of $\mathrm{z}$. The boundary conditions for equation (15) is are discharged from conditions on the upper and lower faces of the wedge. In particular, for the problem $W 1$ matrix A has the form:

$$
\mathbf{A}=\left\|\begin{array}{cc}
0 & 1 \\
S^{2} & -\kappa(\varphi)
\end{array}\right\|, \overrightarrow{\mathbf{x}}=\left\|\begin{array}{c}
W(u, \varphi) \\
W^{\prime}(u, \varphi)
\end{array}\right\|, \kappa(\varphi)=\frac{G^{\prime}(\varphi)}{G(\varphi)}
$$

where prime denotes the derivative with respect $\varphi$. Boundary conditions are: $z_{1}(u, \alpha)=0$, $z_{2}(u, \alpha)=1$.

For the problem $W 2$,

$$
\mathbf{A}=\left\|\begin{array}{cccc}
0 & 1 & 0 & 0 \\
-\frac{2(1-v)}{1-2 v}\left(s^{2}-1\right) & -F_{1}(\varphi) & (s+1) F_{1}(\varphi) & \frac{s+1}{1-2 v}+2 \\
0 & 0 & 0 & 1 \\
F_{2}(\varphi) & \frac{s-3+4 v}{2(1-v)} & -\frac{1-2 v}{2(1-v)}\left(s^{2}-1\right) & -F_{3}(\varphi)
\end{array}\right\|, \overrightarrow{\mathbf{x}}=\left\|\begin{array}{c}
U(u, \varphi) \\
U^{\prime}(u, \varphi) \\
V(u, \varphi) \\
V^{\prime}(u, \varphi)
\end{array}\right\|,
$$

where

$$
\begin{aligned}
& F_{1}(\varphi)=\frac{E^{\prime}}{E}-\frac{v^{\prime}}{v+1}, F_{2}(\varphi)=\frac{E^{\prime}}{E}\left(s \frac{v}{1-v}-1\right)+\frac{v^{\prime}}{1-v^{2}} \frac{s\left(1+2 v^{2}\right)+2 v(v-2)}{1-2 v}, \\
& F_{3}(\varphi)=\frac{E^{\prime}}{E}-\frac{2 v v^{\prime}(v-2)}{\left(1-v^{2}\right)(1-2 v)}, v=v(\varphi), E=E(\varphi)
\end{aligned}
$$

The boundary conditions for this problem are obtained from the conditions in the form (2) and (3).

Further, the solution of the system of differential equations is represented as a linear combination of fundamental solutions

$$
\overrightarrow{\boldsymbol{w}}=\sum_{i=1}^{r} C_{i}(u) \vec{\Psi}_{i}^{F}(u, \varphi),
$$

where $\vec{\Psi}_{i}^{F}(u, \varphi)=\boldsymbol{T}_{i}(u, \varphi) \vec{\Psi}_{i}(u, \varphi), \quad \mathbf{T}_{i}(u, \varphi)$ - diagonal matrices along the diagonal of which are located the components of vectors $\overrightarrow{\boldsymbol{t}}_{i}(u, \varphi)=\left(t_{i}^{1}(u, \varphi), t_{i}^{2}(u, \varphi), \ldots, t_{i}^{r}(u, \varphi)\right)$ Vectors $\vec{\Psi}_{i}(u, \varphi)$ are eigenvectors of matrices $\boldsymbol{A}$ for $\mathrm{k}(\varphi)=0$ (for the case of homogeneous 
medium), $\overrightarrow{\boldsymbol{t}}_{i}(u, \varphi)$ - simulating functions vectors, deal with the medium inhomogeneity, $\mathrm{Ci}(u)$ - some coefficients that are independent of $\varphi$ and determined by solving a system of linear algebraic equations, obtained from the boundary conditions. This method allows us to allocate in eqn. (7) components $\vec{\Psi}_{i}(u, \varphi)$, impeding the process of numerical implementation of solutions. The component type is shown, for example, in [1].

The modeling functions $\overrightarrow{\boldsymbol{t}}_{i}(u, \varphi)$ are determined from the Caughy problem for the fixed values $\mathrm{u}$.

$$
\frac{d \vec{t}_{i}(u, \varphi)}{d \varphi}=\vec{\Psi}_{i}^{-1}\left(A \vec{\Psi}_{i}-\boldsymbol{E} \frac{d \vec{\Psi}_{i}}{d \varphi}\right) \overrightarrow{\boldsymbol{t}}_{i}(u, \varphi) \quad(i=1, \ldots, r), 0 \leq \varphi \leq \alpha
$$

Initial conditions for the Caughy problem are determined from the form of homogeneous solution of (7).

So, the next expressions are were obtained for numerical construction of IE transform functions $\mathrm{L}_{1}(u, \varphi)$ :

$$
\begin{gathered}
(W 1): L_{1}(u, \alpha)=\operatorname{th}(u A \alpha) t_{1}^{1} / t_{1}^{2} \\
(W 2): L_{1}(u, \alpha)=V(i u, \alpha)=a_{1} t_{1}^{3} l+a_{2} t_{2}^{3} k+a_{3} t_{3}^{3} n+a_{4} t_{4}^{3} m, \\
l=\cos (i u+1) \alpha, k=\sin (i u+1) \alpha, n=\cos (i u-1) \alpha, m=\sin (i u-1) \alpha
\end{gathered}
$$

Here $t_{\mathrm{i}}{ }^{3}=t_{\mathrm{i}}{ }^{3}(\mathrm{u}, \varphi)$ are the solutions of Cauchy problems (10), $a_{\mathrm{i}}=a_{\mathrm{i}}(u)-$ solution of the system of linear algebraic equations, obtained from the boundary conditions of the problem (W2).

\subsection{Approximation of the constructed transforms functions by an analytic expression of a special type}

For the considered problems it can be demonstrated similar [5] the kernel transform $L(u)$ of DIE has the following properties under the preceding hypothesis the elastic modules are neither vanish no become infinite.

$$
\begin{gathered}
L(u)=A|u|+B u^{2}+O\left(u^{3}\right), u \rightarrow 0 ; A=\lim _{u \rightarrow 0} L(u) u^{-1} ; \\
L(u)=1+C|u|^{-1}+D u^{-2}+O\left(u^{-3}\right), u \rightarrow \infty
\end{gathered}
$$

The properties of the transformants of the kernels of integral equations established for a multilayered and homogeneous base [1,2] differ from those of the transformants of kernels of integral equations obtained for a gradient wedge.

In $[2,5]$ a theorem is proved that the function $L_{1}(u, \alpha)$, with properties (11) can be approximated by expressions of the form:

$$
L(u)=L_{N}(u)=\tanh A u \prod_{n=1}^{N} \frac{u^{2}+\delta_{n}^{\prime 2}}{u^{2}+\gamma_{n}^{\prime 2}}, \delta_{n}^{\prime}=\delta_{n} \lambda^{-1}, \gamma_{n}^{\prime}=\gamma_{n} \lambda^{-1}
$$

For a numerical realization the improving approximation of $L_{1}(u, \alpha)$ by functions of the type (12) it can be achieved successfully by using the algorithm described in [1]. The integral equation (5) reduces to solving a pairwise integral equation whose solution for the function $L_{1}(u, \alpha)$ of the form (12) is found in the case when the function $g(x)$ can be represented as a Fourier series; the size of the contact zone is fixed and does not depend on the load.

Using the method of [4], an analytical form of the solution is obtained in a form convenient for technical applications, which is expressed in terms of the associated Legendre functions and integrals of them $P_{\mathrm{u}}{ }^{\mu}(\operatorname{ch} \vartheta), Q_{\mathrm{u}}{ }^{\mu}(\operatorname{ch} \vartheta)$, and integrals of them, $\vartheta=\pi /(A \lambda)$, 
$A, \delta_{n}, \gamma_{n}$ - coefficients of approximation of the transformant $L_{1}(u, \alpha)$ by the expression $(10)$ with $N=1$.

where

$$
\begin{aligned}
\phi(x) & =C\left\{\vartheta F_{1}(2(\operatorname{ch} \vartheta-\operatorname{ch} \vartheta x))^{-0,5}\left(Q_{-0,5} L(0)\right)^{-1}+\right. \\
& \left.+\left(F_{2} \delta^{2} \lambda^{-2} \operatorname{sh}^{-1} \vartheta\left(\delta^{2} F_{3}+\gamma^{2} F_{4}\right)^{-1}\right) I(x)\right\}
\end{aligned}
$$

$$
\begin{gathered}
\omega=-0,5+\delta /(\lambda \vartheta), v=-0,5+\gamma /(\lambda \vartheta), F_{1}=1-h(\omega ; 0,5), F_{2}=\left(\delta^{2}-\gamma^{2}\right) Q_{v}, \\
F_{3}=P_{\omega} G(-0,5 ; v), F_{4}=Q_{v} h(\omega ;-0,5), h(a, b)=P_{a}(\operatorname{ch} \vartheta) Q_{b}^{1}(\operatorname{ch} \vartheta)-P_{a}^{1}(\operatorname{ch} \vartheta) Q_{b}(\operatorname{ch} \vartheta), \\
G(a, b)=Q_{a}(\operatorname{ch} \vartheta) Q_{b}^{1}(\operatorname{ch} \vartheta)-Q_{a}^{1}(\operatorname{ch} \vartheta) Q_{b}(\operatorname{ch} \vartheta), \\
I(x)=\int_{x}^{1} P_{\omega}(\operatorname{ch} \vartheta \tau)(2(\operatorname{ch} \vartheta-\operatorname{ch} \vartheta x))^{-0,5} \operatorname{sh} \vartheta \tau d \tau .
\end{gathered}
$$

It is established [4] that the solutions constructed are two-sided asymptotically exact solutions of the original integral equation, both for small and large values of the dimensionless geometric parameter $\lambda$. A comparison of the analytical approximate solutions to the known solutions for a homogeneous wedge of the monograph [1] showed an error of less $1 \%$ for $\lambda<1$ and $\lambda>2.8$. The greatest divergences (more than 10\%) are observed in $\lambda=2$. The explicit analytic form of the solutions of the model provides the possibility of their widespread use for estimating both the mechanical (stiffness function), and protective properties (heat resistance, wear resistance) of functionally gradient coatings of complicated structure.

\section{Numerical results}

\subsection{Numerical examples of constructing transformants of kernels of the integral equation}

In computer simulations, we assume that for all possible types of inhomogeneity the laws of the ratio of Young's modulus on the upper edge of the wedge to the Young's modulus at any interior point (call it $n_{a}$ ) is in the range $1 / 3.5<n_{a}<3.5$. Such properties are described, for example, by composite media $\mathrm{Al}-\mathrm{Fe}, \mathrm{Cr}-\mathrm{Al}, \mathrm{Ag}-\mathrm{Au}$. The analysis is carried out in the case of the law of variation of the Young's modulus with respect to the angular coordinate of the wedge, whose first derivative repeatedly changes sign for any value $\varphi \in[0 ; \pi / 4]$. It's considered the following types of inhomogeneity

$$
\frac{E}{E_{0}}=f_{a}(\varphi)=2.25-1.25 * \cos 8 a \varphi \text { or } \frac{E}{E_{0}}=g_{a}(\varphi)=\frac{1}{f_{a}(\varphi)}, 0<\varphi<\pi / 4
$$

for the Young's modulus (1) and the values of Poisson's ratio in the interval $-1<v<0.5$

Analysis showed that the type (class) of the transformant, and, consequently, the type of solution of contact problems for a heterogeneous wedge, is determined mainly by the properties of the upper layers of the wedge.

\subsection{Numerical examples of constructing the dependence of the shearing force on the displacement of the wedge surface under the stamp}

Figure 2 shows the dependence of the shearing force $P$ on the displacement of the wedge surface under the stamp, related to the corresponding value $P_{0}$ in the homogeneous case and 
character parameter $\lambda$ of the problems for different laws of wedge inhomoeneity. The numbers near the curves correspond to the different inhomogeneity laws (14).
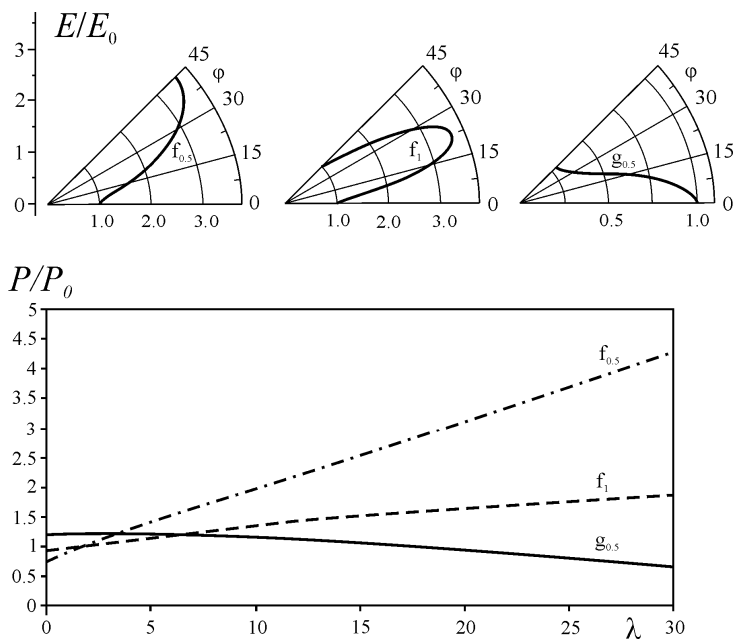

Fig. 2. Relative relationship between inpressing forse $P$ and character parameter $\lambda$ of the problems for different laws of wedge inhomoeneity

\section{Conclusions}

- For sign-alternating laws, in the case of a functionally gradient wedge-shaped coating of an undeformable base, it was possible to construct the simplest analytical solutions of the contact problem, the error of which is less than $10 \%$. These solutions are qualitatively different from solutions for layered materials.

- An approximate analytical solution of the contact problem was obtained in the form convenient for technical applications (determined by three parameters).

- The explicit analytical view of the solutions of this model provides the possibility of their wide use for assessing both the mechanical (stiffness functions) and protective properties (heat resistance, wear resistance) of functional-gradient coatings of a complex structure.

- Numerical analysis of the model shows that the surface properties of the wedge are decisive for the distribution of contact stresses under the die. However, an increase in the number of alternations of soft and hard layers with decreasing wedge solution also has a significant effect on the character of contact stresses.

\section{References}

1. V.M.Alexandrov, Contact tasks in mechanical engineering (Mashinostroenie, Moscow, 1986)

2. S.M.Aizikovich, V.M.Alexandrov, J. J.Kalker, L.I.Krenev, I.S.Trubchik, IJSS, 39(10), 2745 (2002)

3. S.M.Aizikovich, V.M.Alexandrov, I.S.Trubchik, Operator Theory: Advances and Applications, 191, 3 (2009)

4. V.B.Zelentsov, PMM, 46(5), 815 (1982)

5. I.S.Trubchik, L.N.Evich, E.N.Ladosha, AIP Conference Proceedings, 1922, 120011 (2018) 